\title{
Axion phenomenology and lattice QCD
}

\section{Claudio Bonati ${ }^{(a)}$, Massimo D’Elia $^{(a)}$, Marco Mariti $^{(a)}$, G. Martinelli ${ }^{*(b)}$, Michele Mesiti $^{(a)},{\text { Francesco } \operatorname{Negro}^{(a)}, \text { F. Sanfilippo }}^{(c)}$, G. Villadoro ${ }^{(d)}$}

(a) Dipartimento di Fisica dell'Università di Pisa and INFN sezione di Pisa, Largo Pontecorvo 3, I-56127 Pisa, Italy; ${ }^{(b)}$ Dipartimento di Fisica, Università La Sapienza and INFN, Sezione di Roma, Rome, Italy; ${ }^{(c)}$ School of Physics \& Astronomy, University of Southampton, Southampton, United Kingdom. ${ }^{(d)}$ Abdus Salam International Centre for Theoretical Physics, Strada Costiera 11, 34151, Trieste, Italy

The properties of the QCD axion can be studied from the dependence of strong interactions on the $\theta$-parameter. We present a study of the moments of the topological charge for temperatures up to around $600 \mathrm{MeV}$, obtained by lattice QCD simulations with 2+1 flavors at the physical quark masses. Our numerical results for the topological susceptibility, when compared to the instanton gas calculation, differ both in size and in the temperature dependence. We discuss the implications of these results for axion phenomenology, a comparison with other more recent studies and the prospects for future investigations.

34th annual International Symposium on Lattice Field Theory

24-30 July 2016

University of Southampton, $U K$

\footnotetext{
* Speaker.
} 


\section{Introduction}

The axion, advocated as a possible solution to the so-called strong-CP problem through the Peccei-Quinn (PQ) mechanism [1], could also explain the observed dark matter abundance of our Universe [2]. A reliable computation of the axion relic density and of the axion mass requires however a quantitative estimate of the parameters entering the axion effective potential, namely its mass and self-coupling, as a function of the temperature.

Large part of the relevant information can be obtained from the dependence of strong interactions on the topological parameter $\theta$, which enters the QCD Euclidean Lagrangian density as

$$
\mathscr{L}_{\theta}=\frac{1}{4} F_{\mu v}^{a}(x) F_{\mu v}^{a}(x)-i \theta q(x) ; q(x)=\frac{g^{2}}{64 \pi^{2}} \varepsilon_{\mu v \rho \sigma} F_{\mu \nu}^{a}(x) F_{\rho \sigma}^{a}(x)
$$

where $q(x)$ is the topological charge density.

The free energy density of the system can be parametrized as follows

$$
F(\theta, T)=F(0, T)+\frac{1}{2} \chi(T) \theta^{2}\left(1+b_{2}(T) \theta^{2}+b_{4}(T) \theta^{4}+\cdots\right),
$$

where $\chi(T)$ is the topological susceptibility at $\theta=0$, which is proportional to the axion mass

$$
\chi=\int d^{4} x\langle q(x) q(0)\rangle_{\theta=0}=\frac{\left\langle Q^{2}\right\rangle_{\theta=0}}{\mathscr{V}},
$$

where $Q=\int d^{4} x q(x)$ is the global topological charge and $\mathscr{V}=V / T$ is the 4-dimensional volume. The coefficients $b_{n}$ are proportional to the higher cumulants of the topological charge distribution; for instance

$$
b_{2}=-\frac{\left\langle Q^{4}\right\rangle_{\theta=0}-3\left\langle Q^{2}\right\rangle_{\theta=0}^{2}}{12\left\langle Q^{2}\right\rangle_{\theta=0}}
$$

provides information about the axion self interaction.

Analytic predictions about $\chi$ and the $b_{n}$ coefficients are available only in certain regimes. At small (zero) temperature axial transformations can rotate $\theta$ to the light quark sector, allowing to exploit chiral perturbation theory (ChPT) [3]-[4], with the prediction $\chi^{1 / 4}=77.8(4) \mathrm{MeV}$ and $b_{2}=-0.022(1)$ in the case of two degenerate flavors.

At high- $T$, instead, a possible approach is based on the Dilute Instanton Gas Approximation (DIGA). Indeed, since instantons of size $\rho \gg 1 / T$ are suppressed by thermal fluctuations, at asymptotically high- $T$ one can use the perturbative 1-loop estimate for the instanton action, $S_{\text {eff }} \simeq 8 \pi^{2} / g^{2}(T)$, which leads to the prediction of a path integral dominated by a dilute gas of weakly interacting objects of topological charge one. The $\theta$-dependence of the free energy is of the form [5]

$$
F(\theta, T)-F(0, T) \simeq \chi(T)(1-\cos \theta),
$$

and, using the 1-loop effective action, one obtains

$$
\chi(T) \sim T^{4}\left(\frac{m_{l}}{T}\right)^{N_{f}} e^{-8 \pi^{2} / g^{2}(T)} \sim m_{l}^{N_{f}} T^{4-\frac{11}{3} N_{c}-\frac{1}{3} N_{f}}
$$

for $N_{f}$ light flavors of mass $m_{l}$. For example $\chi \propto T^{-7.66}$ for $N_{f}=2$. The range of reliability of the perturbative prediction is not known a priori, and in principle one would trust it only for temperatures much larger than $T_{c} \sim \Lambda_{\mathrm{QCD}}$. 


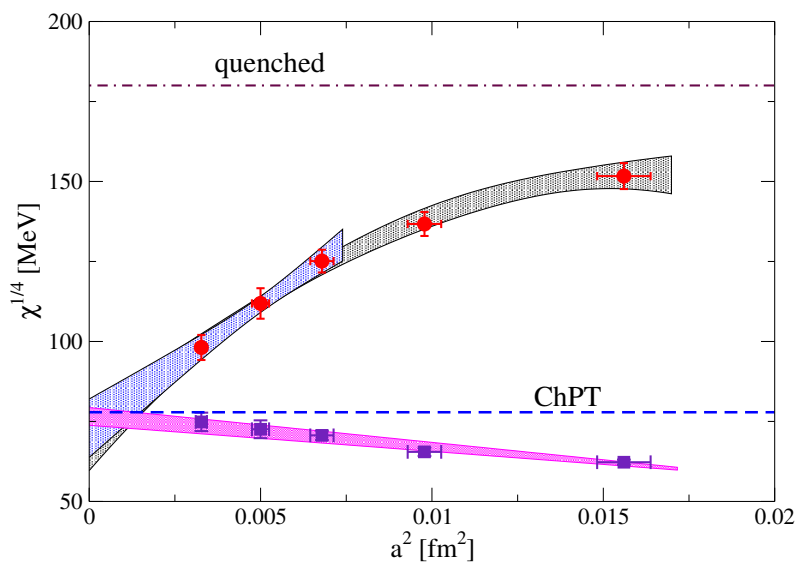

Figure 1: Continuum limit of $\chi$ at $T=0$, compared with the ChPT prediction and the quenched value. Square points correspond to the quantity $\chi_{t c}^{1 / 4}(a)$ (see Eq. 2.1).

A fully non-perturbative determination of $\theta$-dependence can be obtained only by lattice QCD simulations. One needs to implement a lattice discretization of the theory close enough to the continuum limit to reproduce the correct chiral properties of fermion fields. In this limit however the correct sampling of topological modes is affected by critical slowing down as the continuum limit is approached [6]-[7], and is even more difficult in the high $T$ region, where topological fluctuations become very rare.

In the following we present a lattice study of the topological properties of $N_{f}=2+1 \mathrm{QCD}$ with physical quark masses. We adopt stout improved [8] staggered fermions and the tree level improved Symanzik action $[9,10]$ for the pure gauge sector, exploring several lattice spacings, in a range $\sim 0.05-0.12 \mathrm{fm}$, on a line of constant physics, which is fixed following the determinations reported in Refs. [11, 12]; the topological content of the gauge configurations is extracted by a gauge operator after proper smoothing of gauge fields [13]-[14]. More technical details and references can be found in Ref. [15].

In order to identify a proper scaling window and compare the continuum extrapolated results for the topological susceptibility to the ChPT prediction, we first consider simulations at $T=0$. At finite $T$, we explore a region going up to around $4 T_{c}$ and compare our results with DIGA predictions and with other recent results, discussing implications for axion phenomenology and prospects for future investigations.

\section{Numerical Results at Zero and Finite Temperature}

Results obtained for $\chi^{1 / 4}$ at $T=0$, after extrapolation to the infinite volume limit, are reported in Fig. 1 as a function of $a^{2}$, together with the expectation from ChPT. The range of explored lattice spacings is limited at small $a$ by the freezing of the topological modes that we experienced for $a<0.05 \mathrm{fm}$, see Ref. [15].

Finite cut-off effects are significant and for $a \geq 0.1 \mathrm{fm}$ the reported values are closer to the quenched case rather than to the ChPT prediction. Still, we can perform a reliable continuum extrapolation of our data by fitting $O\left(a^{2}\right)$ corrections at the three finest lattice spacings and $O\left(a^{4}\right)$ 


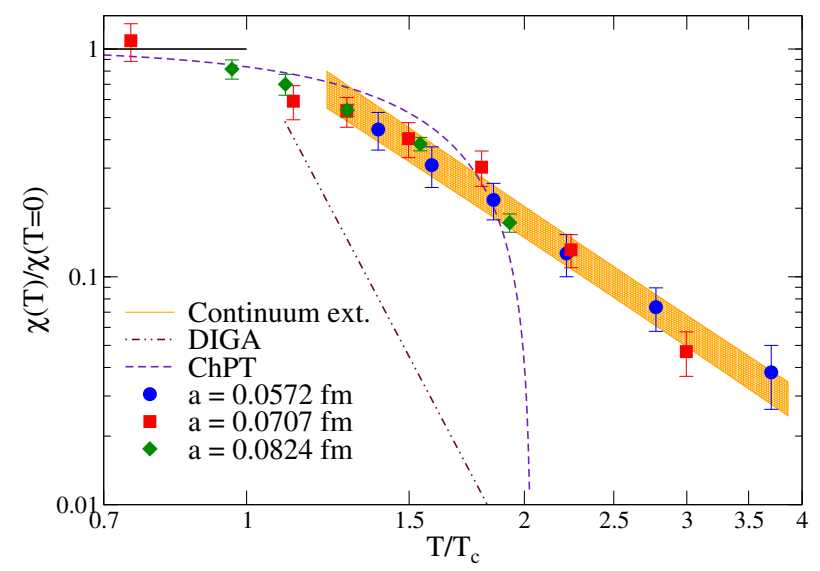

Figure 2: Topological susceptibility, divided by $\chi(T=0)$ at the same lattice spacing, as a function of $T$. The solid line fixes the zero temperature value, the dashed line is the finite $T$ prediction from ChPT, while the dashed-dotted line corresponds to the slope predicted by the dilute instanton gas prediction. The continuum extrapolation has been performed according to Eq. (2.2).

corrections to describe the whole range. We obtain consistent continuum extrapolated results $\left(\chi^{1 / 4}=73(9) \mathrm{MeV}\right.$ in the former case and $\chi^{1 / 4}=69(9) \mathrm{MeV}$ in the latter), which are also in agreement with ChPT.

The large cutoff effects are related to corrections to the chiral properties of light quarks at finite lattice spacing. We tried to reduce these effects by extrapolating the combination

$$
\chi_{t c}^{1 / 4}(a)=a \chi^{1 / 4}(a) \frac{m_{\pi}^{\text {phys }}}{a m_{n g b}(a)}
$$

where $m_{n g b}(a)$ is one of the massive pions found at finite $a$. The values of $\chi_{t c}^{1 / 4}(a)$ are reported in Fig. 1 as square points: in this way cut-off effects are strongly reduced and can be parametrised as $O\left(a^{2}\right)$ corrections over the whole range.

Finite temperature simulations have been performed at the three smallest lattice spacings for which $O\left(a^{2}\right)$ corrections suffice to describe the $T=0$ data, and at various values of the temporal extent $N_{t}$, with a fixed spatial extent $N_{s}=48$, after checking the absence of appreciable finite size effects. Results for the ratio $\chi(T, a) / \chi(T=0, a)$ are reported in Fig. 2 as a function of $T / T_{c}$ $\left(T_{c}=155 \mathrm{MeV}\right)$ together with the DIGA and ChPT predictions. Lattice artifacts seem to be strongly suppressed in this ratio and indeed we found that the dependence on the lattice spacing is quite mild. The difference with the DIGA prediction is evident and indeed a best fit of our data to the function

$$
\frac{\chi(a, T)}{\chi(a, T=0)}=D_{0}\left(1+D_{1} a^{2}\right)\left(\frac{T}{T_{c}}\right)^{D_{2}}
$$

yields a slope coefficient $D_{2} \sim-3$, against a DIGA prediction $D_{2} \sim-8$.

\subsection{Implications for Axion Cosmology}

The low energy effective Lagrangian involving the axion field $a$ has the form

$$
\mathscr{L}_{\text {eff }}=\mathscr{L}_{Q C D}+\frac{1}{2} \partial_{\mu} a \partial^{\mu} a+\left(\theta+\frac{a(x)}{f_{a}}\right) \frac{g^{2}}{32 \pi^{2}} G \tilde{G}+\ldots
$$


where the dots denote other axion interactions. The axion field $a$ has only derivative couplings, apart from the coupling to the QCD topological charge density, regulated by the constant $f_{a}$. By spontaneous breaking of the PQ symmetry, the axion acquires a non-zero vacuum expectation value $\langle a\rangle$. The minimum of the axion effective potential corresponds to a zero effective $\theta$; moreover, assuming that $f_{a}$ is very large, $a$ can be considered as quasi-static and its mass and interaction can be derived from the $\theta$-dependence of QCD. For instance, one has the relation

$$
m_{a}^{2}(T)=\frac{\chi(T)}{f_{a}^{2}}
$$

which would give the value of the axion mass in the present Universe (from the value of $\chi$ at $T=0$ ) as a function of $f_{a}$.

A determination of $f_{a}$ can be obtained by relating the present abundance of axions to the amount of the observed dark matter. One of the best candidates for cosmological axion production is misalignement: at the time when the PQ symmetry breaks, the axion field is not aligned along the minimum, $\theta_{\text {eff }}=0$; the resulting excitation is converted into axion production.

A precise description needs the solution of the axion field evolution which, taking into account only quadratic terms in the axion potential, reads as follows

$$
\ddot{a}(t)+3 H(t) \dot{a}(t)+m_{a}^{2}(t) a(t)=0
$$

where $H$ is the Hubble scale parameter. As the Universe expands and cools down, $H$ decreases as a function of time, while $m_{a}$ increases. Eq. (2.4) predicts a damped solution as long as the friction term wins over the pull from the potential, and becomes oscillatory after the Universe cools below a temperature $T^{*}$ for which $m_{a}\left(T^{*}\right) \approx 3 H\left(T^{*}\right)$. Shortly after that time, the solution develops an adiabatic invariant corresponding to the total number of axions. The value of $T^{*}$ depends both on $\chi(T)$ and on $f_{a}$, so that, by requiring a given amount of axion relics, one can fix the value of $f_{a}$. Actually, an unknown constant is the initial misalignement angle, $\theta_{0}$ : that takes a fixed value throughout the Universe if the PQ symmetry breaking takes place before inflation, otherwise it can acquire all possible values within the visible horizon and one needs to integrate over them.

In Fig. 3, based on the results of our numerical simulations for $\chi(T)$, we show our prediction (see Ref. [15] for more details) for $f_{a}$ and the axion mass, depending on the different possible assumptions, i.e. pre- or post-inflationary scenario and relative amount of dark matter density $\Omega_{D M}$ due to axions from the misalignment mechanism.

Our results lead to values of $f_{a}$ which are typically one order of magnitude larger than those obtained from the dilute instanton gas model, hence to smaller values of the axion mass. This is mostly due to the different behavior of $\chi(T)$, which shows, at least in the explored range, a much milder dependence on $T$ with respect to DIGA predictions. This result might be affected by several systematic effects. In particular, in our study, from results obtained at low temperatures, of order of few hundred $\mathrm{MeV}$, we had to extrapolate to an oscillation temperature of few $\mathrm{GeV}$ and the evolution of the axion field strongly depends on this extrapolation. We thus need a new approach to reach higher temperatures, where at present we do not have results due to the critical slowing down observed at small lattice spacings and to the freezing of the topological charge at higher $T$.

Recent results from different lattice investigations have shown contrasting results. While the results of Ref. [16] are in qualitative agreement with ours, other studies have reported a much better agreement with the instanton gas prediction even in the region right above $T_{c}$ [17]-[19]. 


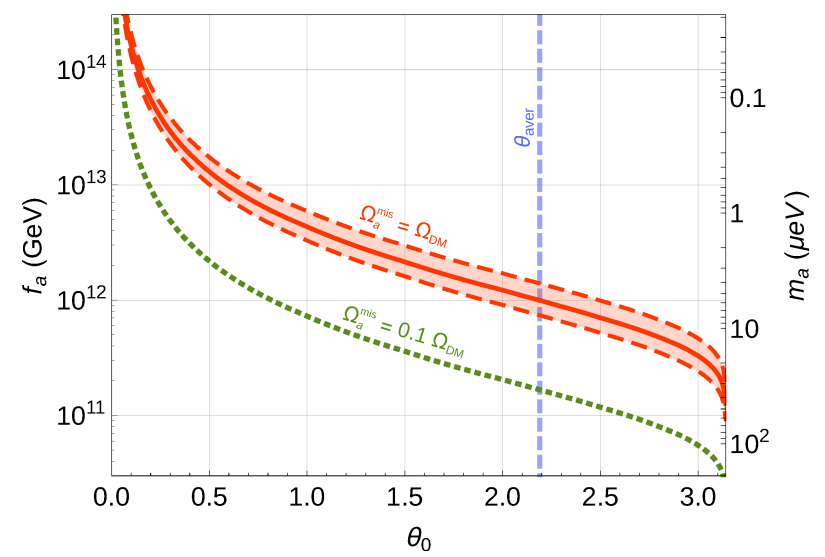

Figure 3: Axion decay constant $f_{a}$ as a function of the angle $\theta_{0}=a_{0} / f_{a}$, such that the misalignment contribution provides one tenth or the whole observed dark matter abundance. The vertical dashed line fixes the average angle $\theta_{\text {aver }}$ for the case in which the PQ symmetry is broken after inflation.

In particular, the authors of Ref. [17] manage to determine $\chi(T)$ for $T$ up to a few GeVs and observe a slope consistent with DIGA shortly after $T_{c}$. In order to reach large values of $T$, without using exceedingly small lattice spacings, they rely on a reweighting procedure of the configurations based on the lowest lying Dirac operator eigenvalues. They also claim that the dynamics of the topological charge and susceptibility at high $\mathrm{T}$ is dominated by only two topological sectors $(Q=$ $0, \pm 1$ ), and restrict the evolution of the system to these sectors working at fixed topology. This approximation is equivalent to the assumption, right from the beginning, that the instanton gas is dilute and non-interacting. There are several other assumptions in their calculation, for example most of the simulations at high $T$ are made with degenerate quarks, with the mass of the strange quark, and then extrapolated to the physical point along lines of constant physics, and they also add an extra feed-back correction for the contribution of the instanton-antinstanton configurations. It is not clear to us to which extent the assumptions made in Ref. [17] are justified and affected the final conclusions. This can only be decided by further investigation at high temperatures, with the help of various improvements that have been proposed especially to deal with the critical slowing down of topological modes [20].

\section{Acknowledgments}

Numerical simulations have been performed on the Blue Gene/Q machine Fermi at CINECA, based on an agreement between INFN and CINECA (under project INFN-NPQCD). FN acknowledges financial support from the INFN SUMA project. Work partially supported by the ERC2011 NEWPHYSICSHPC Grant Agreement Number: 27975, by the ERC-2010 DaMESyFla Grant Agreement Number: 267985, and by the MIUR (Italy) under a contract PRIN10-11 protocollo 2010YJ2NYW.

\section{References}

[1] R. D. Peccei and H. R. Quinn, Phys. Rev. Lett. 38, 1440 (1977) and Phys. Rev. D 16, 1791 (1977). F. Wilczek, Phys. Rev. Lett. 40 (1978) 279; S. Weinberg, Phys. Rev. Lett. 40 (1978) 223. 
[2] J. Preskill, M. B. Wise and F. Wilczek, Phys. Lett. B 120 (1983) 127; L. F. Abbott and P. Sikivie, Phys. Lett. B 120 (1983) 133; M. Dine and W. Fischler, Phys. Lett. B 120 (1983) 137.

[3] P. Di Vecchia and G. Veneziano, Nucl. Phys. B 171 (1980) 253; H. Leutwyler and A. V. Smilga, Phys. Rev. D 46, 5607 (1992); Y. Y. Mao et al. [TWQCD Collaboration], Phys. Rev. D 80 (2009) 034502; F. K. Guo and U. G. Meissner, Phys. Lett. B 749 (2015) 278.

[4] G. G. di Cortona, E. Hardy, J. P. Vega and G. Villadoro, JHEP 1601, 034 (2016).

[5] D. J. Gross, R. D. Pisarski and L. G. Yaffe, Rev. Mod. Phys. 53, 43 (1981); T. Schäfer and E. V. Shuryak, Rev. Mod. Phys. 70, 323 (1998).

[6] B. Alles, G. Boyd, M. D’Elia, A. Di Giacomo and E. Vicari, Phys. Lett. B 389, 107 (1996); L. Del Debbio, H. Panagopoulos and E. Vicari, JHEP 0208, 044 (2002); L. Del Debbio, G. M. Manca and E. Vicari, Phys. Lett. B 594, 315 (2004); S. Schaefer et al. [ALPHA Collaboration], Nucl. Phys. B 845, 93 (2011);

[7] R. Kitano and N. Yamada, JHEP 1510, 136 (2015).

[8] C. Morningstar and M. J. Peardon, Phys. Rev. D 69, 054501 (2004) [hep-lat/0311018].

[9] P. Weisz, Nucl. Phys. B 212, 1 (1983).

[10] G. Curci, P. Menotti and G. Paffuti, Phys. Lett. B 130, 205 (1983) [Erratum-ibid. B 135, 516 (1984)].

[11] Y. Aoki, S. Borsanyi, S. Durr, Z. Fodor, S. D. Katz, S. Krieg and K. K. Szabo, JHEP 0906, 088 (2009) [arXiv:0903.4155 [hep-lat]].

[12] S. Borsanyi, G. Endrodi, Z. Fodor, A. Jakovac, S. D. Katz, S. Krieg, C. Ratti and K. K. Szabo, JHEP 1011, 077 (2010) [arXiv:1007.2580 [hep-lat]];

[13] B. Berg, Phys. Lett. B 104, 475 (1981); Y. Iwasaki and T. Yoshie, Phys. Lett. B 131, 159 (1983); S. Itoh, Y. Iwasaki and T. Yoshie, Phys. Lett. B 147, 141 (1984); M. Teper, Phys. Lett. B 162, 357 (1985); E. -M. Ilgenfritz et al., Nucl. Phys. B 268, 693 (1986).

[14] M. Luscher, Commun. Math. Phys. 293, 899 (2010); M. Luscher, JHEP 1008, 071 (2010), [JHEP 1403, 092 (2014)]; C. Bonati and M. D’Elia, Phys. Rev. D 89, 105005 (2014); K. Cichy, A. Dromard, E. Garcia-Ramos, K. Ottnad, C. Urbach, M. Wagner, U. Wenger and F. Zimmermann, PoS LATTICE 2014, 075 (2014); Y. Namekawa, PoS LATTICE 2014, 344 (2015); C. Alexandrou, A. Athenodorou and K. Jansen, Phys. Rev. D 92, 125014 (2015).

[15] C. Bonati, M. D’Elia, M. Mariti, G. Martinelli, M. Mesiti, F. Negro, F. Sanfilippo and G. Villadoro, JHEP 1603, 155 (2016) [arXiv:1512.06746 [hep-lat]].

[16] A. Trunin, F. Burger, E.-M. Ilgenfritz, M. P. Lombardo and M. Müller-Preussker, arXiv:1510.02265 [hep-lat].

[17] S. Borsanyi et al., Nature 539, no. 7627, 69 (2016) [arXiv:1606.07494 [hep-lat]].

[18] P. Petreczky, H. P. Schadler and S. Sharma, Phys. Lett. B 762, 498 (2016) [arXiv:1606.03145 [hep-lat]].

[19] Y. Taniguchi, K. Kanaya, H. Suzuki and T. Umeda, arXiv:1611.02411 [hep-lat].

[20] P. de Forcrand, J. E. Hetrick, T. Takaishi and A. J. van der Sijs, Nucl. Phys. Proc. Suppl. 63, 679 (1998); M. Luscher and S. Schaefer, JHEP 1107, 036 (2011); A. Laio, G. Martinelli and F. Sanfilippo, JHEP 1607, 089 (2016); M. G. Endres, R. C. Brower, W. Detmold, K. Orginos and A. V. Pochinsky, Phys. Rev. D 92, 114516 (2015); W. Bietenholz, P. de Forcrand and U. Gerber, JHEP 1512, 070 (2015). 